Supplement of Adv. Geosci., 45, 377-382, 2018

https://doi.org/10.5194/adgeo-45-377-2018-supplement

(c) Author(s) 2018. This work is distributed under

the Creative Commons Attribution 4.0 License.

(c) (1)

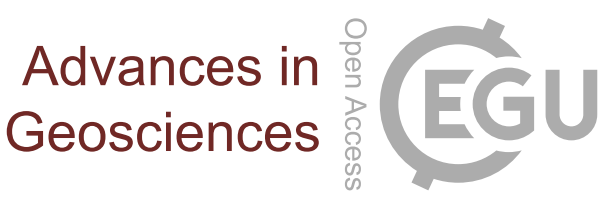

Supplement of

\title{
Accuracy measurement of Random Forests and Linear Regression for mass appraisal models that estimate the prices of residential apartments in Nicosia, Cyprus
}

Thomas Dimopoulos et al.

Correspondence to: Thomas Dimopoulos (thomas.dimopoulos@gmail.com)

The copyright of individual parts of the supplement might differ from the CC BY 4.0 License. 
Figure S1: Distribution of NDEV per Municipality

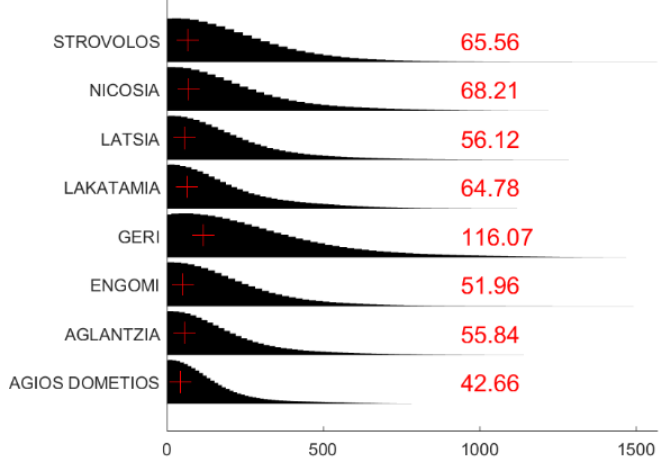

Figure S2: Relieff weights for all the values of DEV

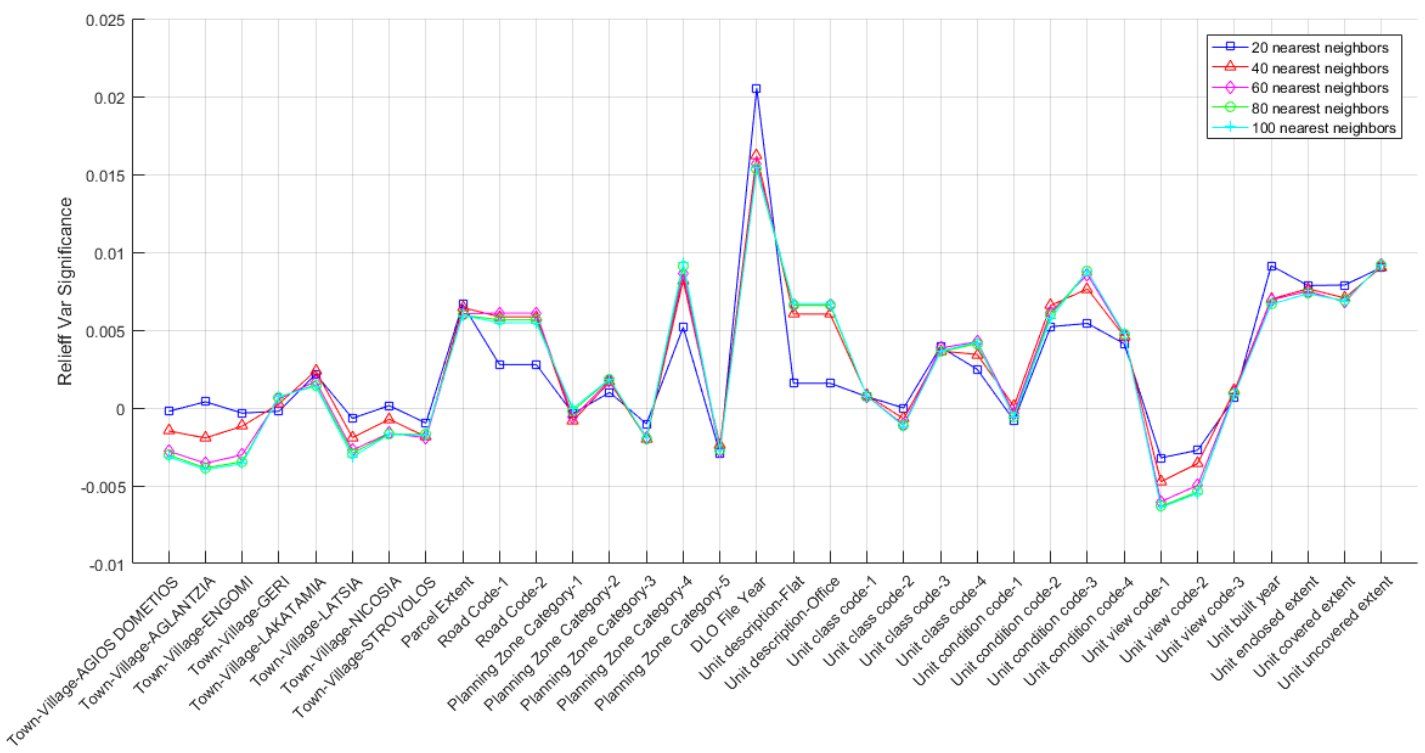

Figure S3: Relieff weights for the non-zero values of DEV

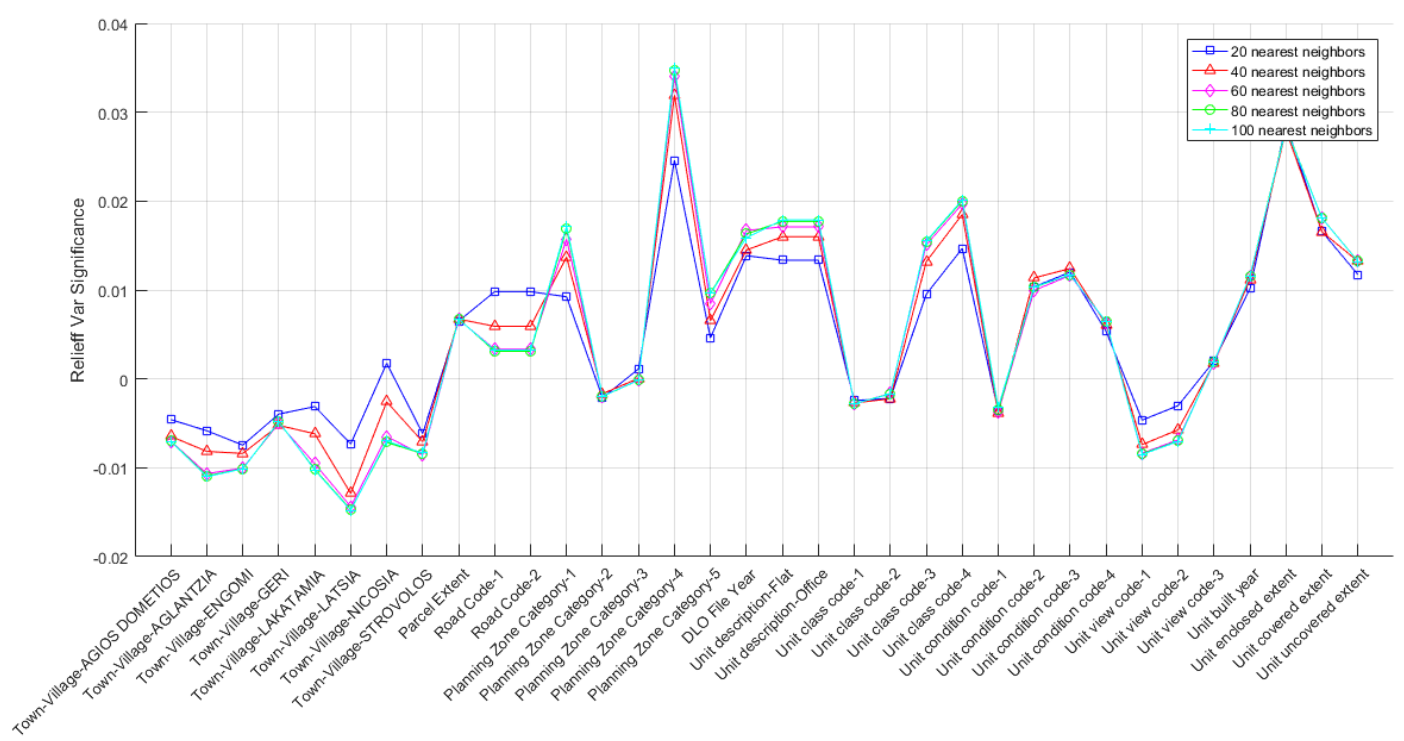


Table S1: Formulas utilized in the models

\begin{tabular}{|c|c|}
\hline Number & Investigated Formula \\
\hline 1 & Accepted_price $\sim$ Town_village_name_mod \\
\hline 2 & Accepted_price $\sim$ Road_code \\
\hline 3 & Accepted_price $\sim$ Shape_code \\
\hline 4 & Accepted_price $\sim$ Unit_class_code \\
\hline 5 & Accepted_price $\sim$ Unit_condition_code \\
\hline 6 & Accepted_price $\sim$ Unit_condition_mod_code \\
\hline 7 & Accepted_price $\sim$ Unit_view_code \\
\hline 8 & Accepted_price $\sim$ Unit_enclosed_extent \\
\hline 9 & Accepted_price $\sim$ Unit_covered_extent \\
\hline 10 & Accepted_price $\sim$ Unit_uncovered_extent \\
\hline 11 & Accepted_price $\sim$ Unit_encl_and_covered_extent \\
\hline 12 & Accepted_price $\sim$ Unit_total_extent \\
\hline 13 & Accepted_price $\sim$ Unit_adjusted_extent \\
\hline 14 & Accepted_price $\sim$ price_index_sale_acceptance_date \\
\hline 15 & Accepted_price $\sim$ flats_index_sale_acceptance_date \\
\hline 16 & Accepted_price $\sim$ Sale_acceptance_year \\
\hline 17 & Accepted_price age_at_sale \\
\hline 18 & Accepted_price $\sim$ Unit_enclosed_extent + Unit_covered_extent \\
\hline 19 & Accepted_price Unit_enclosed_extent + Unit_covered_extent + Unit_uncovered_extent \\
\hline 20 & Accepted_price $\sim$ Unit_enclosed_extent + Unit_covered_extent + Town_village_name_mod \\
\hline 21 & Accepted_price $\sim$ Unit_enclosed_extent + Unit_covered_extent + Unit_class_code \\
\hline 22 & Accepted_price $\sim$ Unit_enclosed_extent + Unit_covered_extent + Unit_condition_code \\
\hline 23 & Accepted_price Unit_enclosed_extent + Unit_covered_extent + Unit_condition_mod_code \\
\hline 24 & Accepted_price $\sim$ Unit_enclosed_extent + Unit_covered_extent + price_index_sale_acceptance_date \\
\hline 25 & Accepted_price Unit_enclosed_extent + Unit_covered_extent + flats_index_sale_acceptance_date \\
\hline 26 & Accepted_price $\sim$ Unit_enclosed_extent + Unit_covered_extent + Sale_acceptance_year \\
\hline 27 & Accepted_price $\sim$ Unit_enclosed_extent + Unit_covered_extent + age_at_sale \\
\hline 28 & Accepted_price $\sim$ Unit_enclosed_extent + Unit_covered_extent + age_at_sale + Town_village_name_mod \\
\hline 29 & Accepted_price $\sim$ Unit_enclosed_extent + Unit_covered_extent + age_at_sale + Unit_class_code \\
\hline 30 & Accepted_price $~$ Unit_enclosed_extent + Unit_covered_extent + age_at_sale + Unit_condition_code \\
\hline 31 & Accepted_price $\sim$ Unit_enclosed_extent + Unit_covered_extent + age_at_sale + Unit_condition_mod_code \\
\hline
\end{tabular}




\begin{tabular}{|c|c|}
\hline 32 & $\begin{array}{l}\text { Accepted_price } \sim \\
\text { price_index_sale_acceptance_date }\end{array}$ \\
\hline 33 & $\begin{array}{l}\text { Accepted_price } \sim \text { Unit_enclosed_extent } \\
\text { flats_index_sale_acceptance_date }\end{array}$ \\
\hline 34 & Accepted_price $\sim$ Unit_enclosed_extent + Unit_covered_extent + age_at_sale + Sale_acceptance_year \\
\hline 35 & $\begin{array}{l}\text { Accepted_price } ~ \text { Unit_enclosed_extent + Unit_covered_extent + age_at_sale + Unit_condition_mod_code } \\
+ \text { Town_village_name_mod }\end{array}$ \\
\hline 36 & $\begin{array}{l}\text { Accepted_price Unit_enclosed_extent + Unit_covered_extent + age_at_sale + Unit_condition_mod_code } \\
+ \text { Unit_class_code }\end{array}$ \\
\hline 37 & $\begin{array}{l}\text { Accepted_price } \sim \text { Unit_enclosed_extent + Unit_covered_extent + age_at_sale + Unit_condition_mod_code } \\
+ \text { price_index_sale_acceptance_date }\end{array}$ \\
\hline 38 & $\begin{array}{l}\text { Accepted_price } ~ \text { Unit_enclosed_extent + Unit_covered_extent + age_at_sale + Unit_condition_mod_code } \\
+ \text { flats_index_sale_acceptance_date }\end{array}$ \\
\hline 39 & $\begin{array}{l}\text { Accepted_price } ~ \text { Unit_enclosed_extent + Unit_covered_extent + age_at_sale + Unit_condition_mod_code } \\
+ \text { Sale_acceptance_year }\end{array}$ \\
\hline 40 & $\begin{array}{l}\text { Accepted_price Unit_enclosed_extent + Unit_covered_extent + age_at_sale + Unit_condition_mod_code } \\
\text { + flats_index_sale_acceptance_date + Town_village_name_mod }\end{array}$ \\
\hline 41 & $\begin{array}{l}\text { Accepted_price } \sim \text { Unit_enclosed_extent + Unit_covered_extent + age_at_sale + Unit_condition_mod_code } \\
+ \text { flats_index_sale_acceptance_date + Unit_class_code }\end{array}$ \\
\hline 42 & $\begin{array}{l}\text { Accepted_price } ~ \text { Unit_enclosed_extent + Unit_covered_extent + age_at_sale + Unit_condition_mod_code } \\
+ \text { flats_index_sale_acceptance_date + Unit_class_code + Town_village_name_mod }\end{array}$ \\
\hline 43 & $\begin{array}{l}\text { Accepted_price } \sim \text { Unit_enclosed_extent }+ \text { Unit_covered_extent + age_at_sale + Unit_condition_mod_code } \\
+\quad \text { flats_index_sale_acceptance_date }+ \text { Unit_class_code } \stackrel{+}{+} \text { Town_village_name_mod }+ \\
\text { Unit_uncovered_extent }\end{array}$ \\
\hline 44 & $\begin{array}{l}\text { Accepted_price } \sim \text { Unit_enclosed_extent }+ \text { Unit_covered_extent + age_at_sale + Unit_condition_mod_code } \\
+\quad \text { flats_index_sale_acceptance_date }+ \text { Unit_class_code } \stackrel{+}{+} \text { Town_village_name_mod }+ \\
\text { Unit_uncovered_extent }+ \text { price_index_sale_acceptance_date }\end{array}$ \\
\hline 45 & $\begin{array}{l}\text { Accepted_price } \sim \text { Unit_enclosed_extent }+ \text { Unit_covered_extent }+ \text { age_at_sale + Unit_condition_mod_code } \\
+\quad \text { flats_index_sale_acceptance_date }+ \text { Unit_class_code }++ \text { Town_village_name_mod }+ \\
\text { Unit_uncovered_extent }+ \text { price_index_sale_acceptance_date }+ \text { Unit_view_code }\end{array}$ \\
\hline
\end{tabular}

Table S2: Accuracy Measures for Linear Regression

\begin{tabular}{|l|l|l|l|l|l|l|}
\hline model & lin_coef & RMSE & MAE & MAPE & sr & method \\
\hline 1 & 0.05 & 53216.5 & 42414.9 & 0.39 & 1.23 & Linear Regression \\
\hline 2 & 0 & 55032 & 44374.4 & 0.4 & 1.24 & Linear Regression \\
\hline 3 & 0 & 54683.4 & 44076.5 & 0.4 & 1.23 & Linear Regression \\
\hline 4 & 0.04 & 52935.8 & 41734.4 & 0.38 & 1.21 & Linear Regression \\
\hline 5 & 0.12 & 51147.5 & 40382.8 & 0.37 & 1.21 & Linear Regression \\
\hline 6 & 0.12 & 51213 & 40508.9 & 0.37 & 1.21 & Linear Regression \\
\hline 7 & 0.05 & 52198.7 & 42011.9 & 0.38 & 1.21 & Linear Regression \\
\hline
\end{tabular}




\begin{tabular}{|c|c|c|c|c|c|c|}
\hline 8 & 0.45 & 43077.7 & 33357 & 0.27 & 1.12 & Linear Regression \\
\hline 9 & 0.33 & 45443.4 & 34504.8 & 0.3 & 1.16 & Linear Regression \\
\hline 10 & 0.07 & 52615.6 & 42011.3 & 0.38 & 1.22 & Linear Regression \\
\hline 11 & 0.53 & 40859.7 & 30740.6 & 0.24 & 1.1 & Linear Regression \\
\hline 12 & 0.51 & 41193.3 & 31209.6 & 0.25 & 1.12 & Linear Regression \\
\hline 13 & 0.52 & 41146.1 & 31448.4 & 0.25 & 1.11 & Linear Regression \\
\hline 14 & 0 & 54683 & 44027.9 & 0.4 & 1.24 & Linear Regression \\
\hline 15 & 0 & 54793.9 & 44097.9 & 0.41 & 1.24 & Linear Regression \\
\hline 16 & 0 & 55198.7 & 44376.3 & 0.41 & 1.24 & Linear Regression \\
\hline 17 & 0.15 & 49313 & 39069.6 & 0.35 & 1.19 & Linear Regression \\
\hline 18 & 0.57 & 39678.8 & 28811.1 & 0.23 & 1.1 & Linear Regression \\
\hline 19 & 0.6 & 38436.2 & 28169.8 & 0.22 & 1.1 & Linear Regression \\
\hline 20 & 0.65 & 37996.5 & 28112 & 0.22 & 1.08 & Linear Regression \\
\hline 21 & 0.55 & 38447.9 & 28313.1 & 0.22 & 1.09 & Linear Regression \\
\hline 22 & 0.65 & 35698.7 & 26481.4 & 0.2 & 1.08 & Linear Regression \\
\hline 23 & 0.65 & 35799.8 & 26539.2 & 0.21 & 1.08 & Linear Regression \\
\hline 24 & 0.59 & 39641.8 & 29273.4 & 0.23 & 1.09 & Linear Regression \\
\hline 25 & 0.58 & 39875 & 29248.4 & 0.23 & 1.1 & Linear Regression \\
\hline 26 & 0.59 & 39614.4 & 29373.6 & 0.23 & 1.1 & Linear Regression \\
\hline 27 & 0.73 & 31621.4 & 23221.8 & 0.18 & 1.06 & Linear Regression \\
\hline 28 & 0.74 & 31232.6 & 22887.1 & 0.18 & 1.06 & Linear Regression \\
\hline 29 & 0.73 & 31702 & 23502.1 & 0.19 & 1.06 & Linear Regression \\
\hline 30 & 0.76 & 31519 & 23399.4 & 0.19 & 1.05 & Linear Regression \\
\hline 31 & 0.75 & 31774 & 23472.9 & 0.19 & 1.06 & Linear Regression \\
\hline 32 & 0.74 & 31920.5 & 23607.3 & 0.19 & 1.06 & Linear Regression \\
\hline 33 & 0.73 & 31708.8 & 23312.6 & 0.18 & 1.06 & Linear Regression \\
\hline 34 & 0.74 & 31603.9 & 23266.5 & 0.18 & 1.05 & Linear Regression \\
\hline 35 & 0.76 & 31547.7 & 23209.7 & 0.18 & 1.06 & Linear Regression \\
\hline 36 & 0.75 & 31927.8 & 23676.7 & 0.19 & 1.06 & Linear Regression \\
\hline 37 & 0.75 & 31974.5 & 23742.5 & 0.19 & 1.06 & Linear Regression \\
\hline 38 & 0.75 & 31767.5 & 23465.3 & 0.19 & 1.06 & Linear Regression \\
\hline 39 & 0.75 & 31776.2 & 23583.4 & 0.18 & 1.05 & Linear Regression \\
\hline 40 & 0.76 & 31512.1 & 23176.3 & 0.18 & 1.06 & Linear Regression \\
\hline 41 & 0.75 & 31918.3 & 23665.7 & 0.19 & 1.06 & Linear Regression \\
\hline 42 & 0.76 & 31667.2 & 23411.6 & 0.19 & 1.06 & Linear Regression \\
\hline 43 & 0.77 & 30868.9 & 23027.4 & 0.18 & 1.06 & Linear Regression \\
\hline 44 & 0.78 & 30732 & 23106.5 & 0.18 & 1.05 & Linear Regression \\
\hline 45 & 0.79 & 30495.7 & 22973.8 & 0.18 & 1.05 & Linear Regression \\
\hline
\end{tabular}

Table S3: Accuracy Measures for the Random Forests Method

\begin{tabular}{|l|l|l|l|l|l|l|}
\hline model & lin_coef & RMSE & MAE & MAPE & sr & method \\
\hline 1 & 0.05 & 53211.2 & 42398.7 & 0.39 & 1.23 & Random Forests \\
\hline
\end{tabular}




\begin{tabular}{|c|c|c|c|c|c|c|}
\hline 2 & 0 & 55001.3 & 44342.1 & 0.4 & 1.24 & Random Forests \\
\hline 3 & 0 & 54648.9 & 44019.9 & 0.4 & 1.23 & Random Forests \\
\hline 4 & 0.04 & 52939.6 & 41736.9 & 0.38 & 1.21 & Random Forests \\
\hline 5 & 0.12 & 51135.3 & 40365.5 & 0.36 & 1.21 & Random Forests \\
\hline 6 & 0.12 & 51232.2 & 40543.9 & 0.37 & 1.21 & Random Forests \\
\hline 7 & 0.05 & 52202.5 & 42018.6 & 0.38 & 1.21 & Random Forests \\
\hline 8 & 0.46 & 46422.5 & 34488.6 & 0.27 & 1.11 & Random Forests \\
\hline 9 & 0.41 & 48011.5 & 36344.7 & 0.31 & 1.16 & Random Forests \\
\hline 10 & 0.09 & 54920.8 & 43351.6 & 0.38 & 1.2 & Random Forests \\
\hline 11 & 0.5 & 45033 & 31782.6 & 0.24 & 1.09 & Random Forests \\
\hline 12 & 0.59 & 45231.8 & 31994.7 & 0.25 & 1.1 & Random Forests \\
\hline 13 & 0.5 & 44896.3 & 33133.9 & 0.26 & 1.1 & Random Forests \\
\hline 14 & 0.01 & 59853.3 & 47559.5 & 0.43 & 1.24 & Random Forests \\
\hline 15 & 0.01 & 56177.9 & 45280 & 0.42 & 1.25 & Random Forests \\
\hline 16 & 0 & 55192.8 & 44356.3 & 0.41 & 1.24 & Random Forests \\
\hline 17 & 0.23 & 62465.3 & 44821 & 0.36 & 1.15 & Random Forests \\
\hline 18 & 0.6 & 44138.9 & 29343 & 0.22 & 1.09 & Random Forests \\
\hline 19 & 0.58 & 38781.9 & 27819.4 & 0.22 & 1.1 & Random Forests \\
\hline 20 & 0.56 & 40920.1 & 27676.9 & 0.22 & 1.1 & Random Forests \\
\hline 21 & 0.48 & 37838.1 & 28108.4 & 0.23 & 1.11 & Random Forests \\
\hline 22 & 0.54 & 37359.4 & 26894.2 & 0.22 & 1.1 & Random Forests \\
\hline 23 & 0.52 & 37173 & 27065.2 & 0.22 & 1.11 & Random Forests \\
\hline 24 & 0.55 & 41474.8 & 28768.3 & 0.22 & 1.09 & Random Forests \\
\hline 25 & 0.55 & 40426.3 & 28831 & 0.22 & 1.09 & Random Forests \\
\hline 26 & 0.52 & 41408.3 & 29358.4 & 0.23 & 1.1 & Random Forests \\
\hline 27 & 0.69 & 30659.6 & 21574.3 & 0.17 & 1.06 & Random Forests \\
\hline 28 & 0.61 & 31166.7 & 21881.3 & 0.17 & 1.07 & Random Forests \\
\hline 29 & 0.57 & 31182.6 & 23234 & 0.19 & 1.09 & Random Forests \\
\hline 30 & 0.57 & 32217.1 & 23674.4 & 0.19 & 1.09 & Random Forests \\
\hline 31 & 0.57 & 32244.9 & 23779 & 0.19 & 1.09 & Random Forests \\
\hline 32 & 0.65 & 30730.7 & 21856 & 0.17 & 1.07 & Random Forests \\
\hline 33 & 0.66 & 29845.2 & 21583.6 & 0.17 & 1.07 & Random Forests \\
\hline 34 & 0.62 & 30843.9 & 22166.5 & 0.18 & 1.08 & Random Forests \\
\hline 35 & 0.55 & 32365.3 & 23636.7 & 0.19 & 1.09 & Random Forests \\
\hline 36 & 0.52 & 32518 & 24520.5 & 0.2 & 1.1 & Random Forests \\
\hline 37 & 0.55 & 32605.8 & 24089.8 & 0.2 & 1.1 & Random Forests \\
\hline 38 & 0.57 & 31605.6 & 23487.6 & 0.19 & 1.09 & Random Forests \\
\hline 39 & 0.54 & 32362.1 & 24101.8 & 0.2 & 1.1 & Random Forests \\
\hline 40 & 0.71 & 29418.4 & 20132.1 & 0.15 & 1.06 & Random Forests \\
\hline 41 & 0.7 & 29499.5 & 20647.8 & 0.16 & 1.06 & Random Forests \\
\hline 42 & 0.69 & 29050 & 20133.3 & 0.15 & 1.06 & Random Forests \\
\hline 43 & 0.7 & 27603.7 & 19760.1 & 0.15 & 1.06 & Random Forests \\
\hline
\end{tabular}




\begin{tabular}{|l|l|l|l|l|l|l|}
\hline 44 & 0.71 & 28122.1 & 19856.6 & 0.15 & 1.06 & Random Forests \\
\hline 45 & 0.71 & 27755.9 & 19723.8 & 0.15 & 1.07 & Random Forests \\
\hline
\end{tabular}

Table S4: Accuracy Measures Comparison

\begin{tabular}{|c|c|c|c|c|c|c|}
\hline model & lin_coef & RMSE & MAE & MAPE & sr & method \\
\hline 1 & 0.000 & 0.010 & 0.038 & 0.000 & 0.000 & comparison \\
\hline 2 & - & 0.056 & 0.073 & 0.000 & 0.000 & comparison \\
\hline 3 & - & 0.063 & 0.128 & 0.000 & 0.000 & comparison \\
\hline 4 & 0.000 & -0.007 & -0.006 & 0.000 & 0.000 & comparison \\
\hline 5 & 0.000 & 0.024 & 0.043 & 2.740 & 0.000 & comparison \\
\hline 6 & 0.000 & -0.037 & -0.086 & 0.000 & 0.000 & comparison \\
\hline 7 & 0.000 & -0.007 & -0.016 & 0.000 & 0.000 & comparison \\
\hline 8 & -2.198 & -7.474 & -3.336 & 0.000 & 0.897 & comparison \\
\hline 9 & -21.622 & -5.496 & -5.194 & -3.279 & 0.000 & comparison \\
\hline 10 & - & -4.287 & -3.140 & 0.000 & 1.653 & comparison \\
\hline 11 & 5.825 & -9.717 & -3.333 & 0.000 & 0.913 & comparison \\
\hline 12 & -14.545 & -9.346 & -2.484 & 0.000 & 1.802 & comparison \\
\hline 13 & 3.922 & -8.717 & -5.220 & -3.922 & 0.905 & comparison \\
\hline 14 & - & -9.028 & -7.712 & -7.229 & 0.000 & comparison \\
\hline 15 & - & -2.494 & -2.645 & -2.410 & -0.803 & comparison \\
\hline 16 & - & 0.011 & 0.045 & 0.000 & 0.000 & comparison \\
\hline 17 & -42.105 & -23.533 & -13.712 & -2.817 & 3.419 & comparison \\
\hline 18 & -5.128 & -10.642 & -1.829 & 4.444 & 0.913 & comparison \\
\hline 19 & 3.390 & -0.895 & 1.251 & 0.000 & 0.000 & comparison \\
\hline 20 & 14.876 & -7.409 & 1.560 & 0.000 & -1.835 & comparison \\
\hline 21 & 13.592 & 1.599 & 0.725 & -4.444 & -1.818 & comparison \\
\hline 22 & 18.487 & -4.546 & -1.547 & -9.524 & -1.835 & comparison \\
\hline 23 & 22.222 & -3.764 & -1.963 & -4.651 & -2.740 & comparison \\
\hline 24 & 7.018 & -4.519 & 1.740 & 4.444 & 0.000 & comparison \\
\hline 25 & 5.310 & -1.373 & 1.437 & 4.444 & 0.913 & comparison \\
\hline 26 & 12.613 & -4.428 & 0.052 & 0.000 & 0.000 & comparison \\
\hline 27 & 5.634 & 3.088 & 7.355 & 5.714 & 0.000 & comparison \\
\hline 28 & 19.259 & 0.211 & 4.493 & 5.714 & -0.939 & comparison \\
\hline 29 & 24.615 & 1.652 & 1.147 & 0.000 & -2.791 & comparison \\
\hline 30 & 28.571 & -2.191 & -1.169 & 0.000 & -3.738 & comparison \\
\hline 31 & 27.273 & -1.471 & -1.296 & 0.000 & -2.791 & comparison \\
\hline 32 & 12.950 & 3.798 & 7.704 & 11.111 & -0.939 & comparison \\
\hline 33 & 10.072 & 6.055 & 7.702 & 5.714 & -0.939 & comparison \\
\hline 34 & 17.647 & 2.434 & 4.842 & 0.000 & -2.817 & comparison \\
\hline 35 & 32.061 & -2.559 & -1.823 & -5.405 & -2.791 & comparison \\
\hline 36 & 36.220 & -1.832 & -3.501 & -5.128 & -3.704 & comparison \\
\hline 37 & 30.769 & -1.955 & -1.452 & -5.128 & -3.704 & comparison \\
\hline
\end{tabular}




\begin{tabular}{|l|l|l|l|l|l|l|}
\hline 38 & 27.273 & 0.511 & -0.095 & 0.000 & -2.791 & comparison \\
\hline 39 & 32.558 & -1.827 & -2.174 & -10.526 & -4.651 & comparison \\
\hline 40 & 6.803 & 6.873 & 14.058 & 18.182 & 0.000 & comparison \\
\hline 41 & 6.897 & 7.876 & 13.621 & 17.143 & 0.000 & comparison \\
\hline 42 & 9.655 & 8.621 & 15.057 & 23.529 & 0.000 & comparison \\
\hline 43 & 9.524 & 11.168 & 15.272 & 18.182 & 0.000 & comparison \\
\hline 44 & 9.396 & 8.869 & 15.129 & 18.182 & -0.948 & comparison \\
\hline 45 & 10.667 & 9.407 & 15.224 & 18.182 & -1.887 & comparison \\
\hline \%diff & $\mathbf{9 . 7 3 0 7 6 5}$ & $\mathbf{- 1 . 2 7 1 7 9}$ & $\mathbf{1 . 4 4 3 6 7 3}$ & $\mathbf{2 . 0 7 2 5 1 8}$ & $-\mathbf{- 0 . 7 3 4 3 1}$ & \\
\hline
\end{tabular}

Table S5: Description of the fields in the studied Database

\begin{tabular}{|c|c|c|c|}
\hline$A$ & Sbpi_id_no & $\begin{array}{l}\text { The unique identification number } \\
\text { for the CILIS database (GIS). }\end{array}$ & Integer \\
\hline$B$ & District_name & The district name. & String \\
\hline $\mathrm{C}$ & Town_village_name & $\begin{array}{l}\text { It identifies the municipality / } \\
\text { village. }\end{array}$ & String \\
\hline $\mathrm{D}$ & Quarter_name & $\begin{array}{l}\text { Separation of municipalities into } \\
\text { quarters. The separation is based } \\
\text { on the closest church (Parish) }\end{array}$ & String \\
\hline$E$ & District & $\begin{array}{l}\text { The district name of the variable } \\
\text { District_name is coded } \\
\text { numerically. }\end{array}$ & Integer \\
\hline $\mathrm{F}$ & Town_village & $\begin{array}{l}\text { The municipality/village name of } \\
\text { the variable Town_village_name is } \\
\text { coded numerically. }\end{array}$ & Integer \\
\hline G & Quarter & $\begin{array}{l}\text { The quarter name of the variable } \\
\text { Quarter_name is coded } \\
\text { numerically. }\end{array}$ & Integer \\
\hline $\mathrm{H}$ & Block & The plans are divided into plots. & Integer \\
\hline 1 & Reg_no & The Unique registration number. & Integer \\
\hline $\mathrm{J}$ & Sheet & $\begin{array}{l}\text { Cyprus cadastral map is divided in } \\
60 \text { Sheets (scale 1:5000, } 2 \text { inches = } \\
1 \text { mile). }\end{array}$ & Integer \\
\hline $\mathrm{K}$ & Plan & $\begin{array}{l}\text { Every sheet is further divided into } \\
4 \text { plans or smaller (scales 1:2500, } \\
\text { 1:1000, 1:500). }\end{array}$ & String \\
\hline $\mathrm{L}$ & Parcel_no & $\begin{array}{l}\text { The parcel number. Parcel (or plot) } \\
\text { is the minimum division. }\end{array}$ & Integer \\
\hline$M$ & Parcel_extent & Area of the parcel (or plot). & Double \\
\hline $\mathrm{N}$ & Access_code & $\begin{array}{l}\text { It denotes the access to the parcel. } \\
\text { Values: } 1 \text { = access through public } \\
\text { road. Since all data are referring to } \\
\text { developed land, all of them should } \\
\text { be accessible. }\end{array}$ & Integer \\
\hline
\end{tabular}




\begin{tabular}{|c|c|c|c|}
\hline $\mathrm{O}$ & Road_code & $\begin{array}{l}\text { The importance of the adjacent } \\
\text { roads. Values: From } 1 \text { (most } \\
\text { important) to } 2 \text { (less important). }\end{array}$ & Integer \\
\hline$P$ & Shape_code & $\begin{array}{l}\text { It denotes the quality of the shape } \\
\text { of the parcel. Values: From } 1 \text { (best } \\
\text { shape) to } 3 \text { (worst shape). }\end{array}$ & Integer \\
\hline$Q$ & Planning_zone_category & $\begin{array}{l}\text { Values: } 1=\text { residential, } 2=\text { mixed } \\
\text { use (offices and residential), } 3= \\
\text { core (continuous density old city } \\
\text { centres), } 4=\text { commercial and } \\
\text { bussines district, } 5=\text { commercial, } 8 \\
=\text { industrial. }\end{array}$ & Integer \\
\hline $\mathrm{R}$ & Planning_zone_code & The code of the planning zone. & Integer \\
\hline $\mathrm{S}$ & Planning_zone_name & $\begin{array}{l}\text { The name of the planning zone. It } \\
\text { corresponds to the } \\
\text { Planning_zone_code variable. }\end{array}$ & String \\
\hline $\mathrm{T}$ & $\begin{array}{l}\text { Secondary_planning_zone } \\
\text { _category }\end{array}$ & $\begin{array}{l}\text { A secondary category for the } \\
\text { planning zone, in case the parcel } \\
\text { abuts in } 2 \text { different zones. Values: } \\
1=\text { residential, } 2 \text { = mixed use } \\
\text { (offices and residential), } 3 \text { = core } \\
\text { (continuous density old city } \\
\text { centres), } 4=\text { commercial and } \\
\text { bussines district, } 5 \text { = commercial. }\end{array}$ & Integer \\
\hline$U$ & Secondary_planning_zone_code & $\begin{array}{l}\text { The code of the secondary } \\
\text { planning zone in case the parcel } \\
\text { abuts in } 2 \text { different zones. }\end{array}$ & Integer \\
\hline $\mathrm{V}$ & $\begin{array}{l}\text { Secondary_planning_zone } \\
\text { _name }\end{array}$ & $\begin{array}{l}\text { The name of the secondary } \\
\text { planning zone. It corresponds to } \\
\text { the } \\
\text { Secondary_planning_zone_code } \\
\text { variable. }\end{array}$ & String \\
\hline $\mathrm{W}$ & Declared_price & $\begin{array}{l}\text { The corresponding amount (in } € \text { ) } \\
\text { that the buyer and seller declared } \\
\text { at the Cyprus Department of Lands } \\
\text { and Surveys (DLS) for the property } \\
\text { exchange at the transaction date. } \\
\text { No changes made for inflation, } \\
\text { property cycles and/or fluctuation. }\end{array}$ & Double \\
\hline
\end{tabular}




\begin{tabular}{|c|c|c|c|}
\hline$x$ & Accepted_price & $\begin{array}{l}\text { The desktop valuation (in } € \text { ) of DLS } \\
\text { (transaction fees and taxes are } \\
\text { paid on this figure). Theoretically, } \\
\text { this figure must be more accurate } \\
\text { than the Declared_price. However } \\
\text { due to the fact that the DLS is } \\
\text { doing retrospective research, } \\
\text { when property prices are } \\
\text { increasing they are not up to date } \\
\text { (they evaluate a bit lower than real } \\
\text { prices). On the contrary when } \\
\text { property market is not performing } \\
\text { well, when they look back they } \\
\text { have higher figures than the real } \\
\text { ones. No changes made for } \\
\text { inflation, property cycles and/or } \\
\text { fluctuation. }\end{array}$ & Double \\
\hline$Y$ & DLO_file & $\begin{array}{l}\text { All transactions are marked with } \\
\text { Greek Letter " } \Pi \text { " and the sale } \\
\text { number (see } Z \text { column). }\end{array}$ & String \\
\hline Z & DLO_file_no & $\begin{array}{l}\text { The number of the sale (like a } \\
\text { receipt). }\end{array}$ & Integer \\
\hline AA & DLO_file_year & $\begin{array}{l}\text { The year the transaction } \\
\text { happened. }\end{array}$ & Integer \\
\hline$A B$ & Fiscal_property_type & $\begin{array}{l}\text { Horizontal (applicable mainly to } \\
\text { apartment buildings). It applies } \\
\text { when there is ownership of } \\
\text { separate parts of a multi-storey } \\
\text { building. }\end{array}$ & String \\
\hline$A C$ & Sale_acceptance_date & $\begin{array}{l}\text { The date that transaction was } \\
\text { accepted from DLS. }\end{array}$ & Date \\
\hline$A D$ & Share_numerator & $\begin{array}{l}\text { Since the sample under study } \\
\text { consists of flats, the majority of } \\
\text { observations are referring to } 100 \% \\
\text { shares. Those observations that } \\
\text { are not } 100 \% \text { share are excluded } \\
\text { from the sample because a } 50 \% \\
\text { share of a flat does not represent a } \\
\text { rational market value. }\end{array}$ & Integer \\
\hline $\mathrm{AE}$ & Share_denominator & $\begin{array}{l}\text { In case of share. See also column } \\
A D\end{array}$ & Integer \\
\hline $\mathrm{AF}$ & COS_agreement_date & $\begin{array}{l}\text { The date that the involved parties } \\
\text { submitted the contract to the DLS. }\end{array}$ & Date \\
\hline AG & Remark & DLS notes. & String \\
\hline
\end{tabular}




\begin{tabular}{|c|c|c|c|}
\hline $\mathrm{AH}$ & Sales_remark & $\begin{array}{l}\text { DLS notes in Greek. In some cases, } \\
\text { they repeat the declared price or } \\
\text { the covered area. }\end{array}$ & String \\
\hline $\mathrm{Al}$ & Main_sbp_cat & Notes of the DLS. & String \\
\hline AJ & Main_sbp_kind & The type of property. & String \\
\hline AK & Status & All observations are marked as L. & String \\
\hline $\mathrm{AL}$ & Main_sbpi_id_no & $\begin{array}{l}\text { A code from the DLS system } \\
\text { corresponding to the shapefile. }\end{array}$ & Integer \\
\hline AM & Building_code & DLS internal reference. & Integer \\
\hline AN & Building_desc & DLS internal reference. & String \\
\hline $\mathrm{AO}$ & Building_sbpi_id_no & DLS internal reference. & Integer \\
\hline$A P$ & Field42 & DLS internal reference. & String \\
\hline $\mathrm{AQ}$ & Unit_code & $\begin{array}{l}\text { The unit code that corresponds to } \\
\text { the Unit_desc variable in column } \\
\text { AR. Values: } 215=\text { apartment, } 818= \\
\text { coffee shop, } 814=\text { exposition } \\
\text { centre, } 823=\text { health clinic, } 815= \\
\text { laboratory, } 810=\text { office, } 811= \\
\text { office complex, } 813=\text { office with } \\
\text { middle floor, } 837=\text { power station, } \\
853=\text { residence, } 474=\text { shed, } 808= \\
\text { shop, } 812=\text { shop with middle } \\
\text { floor, } 809=\text { shopping complex, } 927 \\
=\text { small industry, } 849=\text { storage, } \\
932=\text { three storey residence, } 824= \\
\text { treatment room, } 232=\text { two storey } \\
\text { apartment, } 861=\text { two storey } \\
\text { building, } 803=\text { two storey } \\
\text { residence, } 805=\text { two storey } \\
\text { residence with garden, } 237= \\
\text { unfinished building. }\end{array}$ & Integer \\
\hline AR & Unit_desc & $\begin{array}{l}\text { The type of property. Values: } \\
\text { apartment, coffee shop, exposition } \\
\text { center, health clinic, laboratory, } \\
\text { office, office complex, office with } \\
\text { middle floor, power station, } \\
\text { residence, shed, shop, shop with } \\
\text { middle floor, shopping complex, } \\
\text { small industry, storage, three } \\
\text { storey residence, treatment room, } \\
\text { two storey apartment, two storey } \\
\text { building, two storey residence, two } \\
\text { storey residence with garden, } \\
\text { unfinished building. }\end{array}$ & String \\
\hline AS & Unit_sbpi_id_no & $\begin{array}{l}\text { A code from the DLS system } \\
\text { corresponding to the shapefile. }\end{array}$ & Integer \\
\hline
\end{tabular}




\begin{tabular}{|l|l|l|l|} 
AT & Field46 & Notes of the DLS. & String \\
\hline AU & Unit_class_code & $\begin{array}{l}\text { It denotes the class of the building. } \\
\text { Values: From 1 (best class) to 4 } \\
\text { (worst class). }\end{array}$ & Integer \\
\hline AV & Unit_condition_code & $\begin{array}{l}\text { It denotes the condition of the } \\
\text { building. Values: From 1 (best } \\
\text { condition) to 4 (worst condition). }\end{array}$ & Integer \\
\hline AW & Unit_view_code & $\begin{array}{l}\text { It denotes the view of the unit. } \\
\text { Values: From 1 (best view) to 4 } \\
\text { (worst view). }\end{array}$ & Integer \\
\hline AX & Unit_built_year & $\begin{array}{l}\text { The date the building was } \\
\text { constructed. }\end{array}$ & Date \\
\hline AY & Unit_enclosed_extent & Internal area (in m2). & Integer \\
\hline AZ & Unit_covered_extent & Area of covered verandahs (in m2). & Integer \\
\hline BA & Unit_uncovered_extent & $\begin{array}{l}\text { Area of uncovered verandahs (in } \\
\text { m2). }\end{array}$ & Integer \\
\hline BB & Total_value_2013 & $\begin{array}{l}\text { The new general valuation (in } € \text { ) } \\
\text { dated the 1st of January 2013. } \\
\text { These values represent the } \\
\text { outcome of the new general } \\
\text { valuation made by the DLS. }\end{array}$ & Integer \\
\hline
\end{tabular}

